\title{
The mathematical modeling of the atmospheric diffusion equation
}

\author{
Khaled Sadek Mohamed Essa ${ }^{1}$, Mohamed Magdy Abd El-Wahab ${ }^{2}$, Hussein Mahmoud ELsman ${ }^{3}$, \\ Adel Shahta Soliman ${ }^{4}$, Samy Mahmoud ELGmmal ${ }^{3}$, Aly Ahamed Wheida ${ }^{4}$ \\ ${ }^{1}$ Department of Mathematics and Theoretical Physics, Nuclear Research Centre, Cairo, Egypt \\ ${ }^{2}$ Astronomy Department, Faculty of Science, Cairo University, Cairo, Egypt \\ ${ }^{3}$ Physics Department, Faculty of science, Monofia University, Monofia, Egypt \\ ${ }^{4}$ Theoretical Physics Department, National Research Centre, Cairo, Egypt
}

Email address:

mohamedksm56@yahoo.com (K. S. M. Essa)

\section{To cite this article:}

Khaled Sadek Mohamed Essa, Mohamed Magdy Abd El-Wahab, Hussein Mahmoud ELsman, Adel Shahta Soliman, Samy Mahmoud ELGmmal, Aly Ahamed Wheida. The Mathematical Modeling of the Atmospheric Diffusion Equation. International Journal of Environmental Monitoring and Analysis. Vol. 2, No. 2, 2014, pp. 112-116. doi: 10.11648/j.ijema.20140202.18

\begin{abstract}
The advection diffusion equation (ADE) is solved in two directions to obtain the crosswind integrated concentration. The solution is solved using separation variables technique and considering the wind speed depends on the vertical height and eddy diffusivity depends on downwind and vertical distances. Comparing between the two predicted concentrations and observed concentration data are taken on the Copenhagen in Denmark.
\end{abstract}

Keywords: Advection Diffusion Equation, Predicted Normalized Crosswind Integrated Concentrations, Separation Variables

\section{Introduction}

The analytical solution of the atmospheric diffusion equation has been containing different shaped depending on Gaussian and non-Gaussian solutions. An analytical solution with power law for the wind speed and eddy diffusivity with the realistic assumption was studied by [1] the solution has been implemented in the KAPPA-G model [2], and [3] extended the solution of [1] under boundary conditions suitable for dry deposition at the ground. The mathematics of atmospheric dispersion modeling is studied by [4]. In the analytical solutions of the diffusion-advection equation, assuming constant along the whole planetary boundary layer (PBL) or following a power law was studied by [5-7], [2] and [8].

Estimating of crosswind integrated Gaussian and non-Gaussian concentration through different dispersion schemes is studied by [9]. Analytical solution of diffusion equation in two dimensions using two forms of eddy diffusivities is studied by [9]

In this paper the advection diffusion equation (ADE) is solved in two directions to obtain crosswind integrated ground level concentration in unstable conditions. We use separation variables technique and considering the wind speed and eddy diffusivity depends on the vertical height and downwind distance. We compare between observed data from Copenhagen (Denmark) and predicted concentration data using statistical technique.

\section{Analytical Method}

Time dependent advection - diffusion equation is written as [10]

$$
\frac{\partial C(x, y, z)}{\partial t}+u \frac{\partial C(x, y, z)}{\partial x}=\frac{\partial}{\partial x}\left(K_{x} \frac{\partial C(x, y, z)}{\partial x}\right)+\frac{\partial}{\partial y}\left(K_{y} \frac{\partial C(x, y, z)}{\partial y}\right)+\frac{\partial}{\partial z}\left(K_{z} \frac{\partial C(x, y, z)}{\partial z}\right)
$$

For steady state, taking $\frac{\partial C(x, y, z)}{\partial t}=0$ and the diffusion in the $\mathrm{x}$-axis direction is assumed to be zero compared with the adjective in the same directions, hence:

$$
u \frac{\partial C(x, y, z)}{\partial x}=\frac{\partial}{\partial y}\left(K_{y} \frac{\partial C(x, y, z)}{\partial y}\right)+\frac{\partial}{\partial z}\left(K_{z} \frac{\partial C(x, y, z)}{\partial z}\right)
$$

where: $C(x, y, z)$ is the average concentration of air pollution $\left(\mu \mathrm{g} / \mathrm{m}^{3}\right)$ in three dimension. $\mathrm{u}$ is the mean wind speed in $\mathrm{x}$-direction $(\mathrm{m} / \mathrm{s})$. 
$K_{y}$ and $K_{z}$ are the eddy diffusivities coefficients which are function in $\mathrm{x}$-direction $\left(\mathrm{m}^{2} / \mathrm{s}\right)$ i,e

$$
K_{x}=K_{y}=K_{z} .
$$

Integrating the equation (1) with respect to $y$ from $-\infty$ to $\infty$ at a point $(x, z)$ of the atmospheric advection-diffusion equation is written in the form [11];

$$
\begin{gathered}
u \frac{\partial}{\partial x} \int_{-\infty}^{\infty} C(x, y, z) d y=K_{y} \frac{\partial C(x, y, z)}{\partial y}+K_{z} \frac{\partial^{2}}{\partial z^{2}}\left(\int_{-\infty}^{\infty} \partial C(x, y, z) d y\right) \\
\text { Let } C_{y}(x, z)=\int_{-\infty}^{\infty} C(x, y, z) d y
\end{gathered}
$$

where $C_{y}(x, z)$ is the normalized crosswind integrated concentration. Note that the value of concentration tends to zero at far distance i.e. $K_{y} \frac{\partial C(x, y, z)}{\partial y}=0$ (4)

Substituting by equations (3) and (4) in Eq. (2) it was getting:

$$
\begin{gathered}
u \frac{\partial C_{y}(x, z)}{\partial x}=\frac{\partial}{\partial z} k_{z}(x) \frac{\partial C_{y}(x, z)}{\partial z} \\
u \frac{\partial C_{y}(x, z)}{\partial x}=k_{z}(x) \frac{\partial^{2} C_{y}(x, z)}{\partial z^{2}}
\end{gathered}
$$

let the solution of Eq. (6) in the form:

$$
C_{y}(x, z)=\sum_{n=0}^{\infty} X_{n}(x) Z_{n}(z)
$$

For simplicity Eq. (7) can be written in the form

$$
C_{y}(x, z)=X_{n}(x) Z_{n}(z)
$$

Substituting by Eq. (8) in Eq. (6) it was getting:

$$
u Z_{n}(z) \frac{d X_{n}(x)}{d x}=k_{z}(x) X_{n}(x) \frac{d^{2} Z_{n}(z)}{d z^{2}}
$$

Dividing Eq. (9) by $k_{z}(x) X_{n}(x) Z_{n}(z)$ then:

$$
\frac{u}{k_{z}(x)} \frac{1}{X_{n}(x)} \frac{d X_{n}(x)}{d x}=\frac{1}{Z_{n}(z)} \frac{d^{2} Z_{n}(z)}{d z^{2}}
$$

Let

$$
\begin{gathered}
\frac{u}{k_{z}(x)} \frac{1}{X_{n}(x)} \frac{d X_{n}(x)}{d x}=\frac{1}{Z_{n}(z)} \frac{d^{2} Z_{n}(z)}{d z^{2}}=-\beta_{n}^{2} \\
\frac{u}{k_{z}(x)} \frac{1}{X_{n}(x)} \frac{d X_{n}(x)}{d x}=-\beta_{n}^{2}
\end{gathered}
$$

And

$$
\frac{1}{Z_{n}(z)} \frac{d^{2} Z_{n}(z)}{d z^{2}}=-\beta_{n}^{2}
$$

$$
\frac{1}{X_{n}(x)} \frac{d X_{n}(x)}{d x}=-\beta_{n}^{2} \frac{k_{z}(x)}{u}
$$

Then

$$
\begin{gathered}
\frac{d X_{n}(x)}{X_{n}(x)}=-\beta_{n}^{2} \frac{k_{z}(x)}{u} d x \\
\Rightarrow \ln X_{n}(x)=\frac{-\beta_{n}^{2}}{u} \int_{0}^{x} k_{z}\left(x^{\prime}\right) d x^{\prime}+\ln \alpha_{n} \\
\Rightarrow \ln X_{n}(x)-\ln \alpha_{n}=\frac{-\beta_{n}^{2}}{u} \int_{0}^{x} k_{z}\left(x^{\prime}\right) d x^{\prime} \\
\ln X_{n}(x)-\ln \alpha_{n}=\frac{-\beta_{n}^{2}}{u} \int_{0}^{x} k_{z}\left(x^{\prime}\right) d x^{\prime} \Rightarrow \ln \frac{X_{n}(x)}{\alpha_{n}}=\frac{-\beta_{n}^{2}}{u} \int_{0}^{x} k_{z}\left(x^{\prime}\right) d x^{\prime} \\
\Rightarrow X_{n}(x)=\alpha_{n} e^{\frac{-\beta_{n}^{2}}{u} \int_{0}^{x}{ }_{0}^{k}(x)(x) \alpha^{\prime}}
\end{gathered}
$$

Eq. (13) can be solved as following:

$$
\frac{d^{2} Z_{n}(z)}{d z^{2}}+\beta_{n}^{2} Z_{n}(z)=0
$$

Eq (18) is simple harmonic equation has solution in the form:

$$
Z_{n}(z)=a_{n} \sin \left(\beta_{n} z\right)+b_{n} \cos \left(\beta_{n} z\right)
$$

Equation (6) is subjected to the following boundary conditions:

1-It is assumed that the pollutants are reflected at the ground surface i.e

$$
\frac{\partial \mathrm{C}_{\mathrm{y}}(\mathrm{x}, \mathrm{z})}{\partial \mathrm{z}}=0 \text { at } \mathrm{z}=0
$$

The flux at the top the mixing layer can be given by

$$
\frac{\partial \mathrm{C}_{\mathrm{y}}(\mathrm{x}, \mathrm{z})}{\partial \mathrm{z}}=0 \text { at } \mathrm{z}=h
$$

where $\mathrm{h}$ is the mixing height.

The mass continuity is written in the form:

$$
u C_{y}(x, z)=Q \delta\left(z-h_{s}\right) \text { at } x=0
$$

where $Q$ is the source strength, $\delta$ is Dirac delta function and $h_{s}$ is the stake height. Then the general solution becomes:

$$
c_{y}(x, z)=\alpha_{n} e^{\frac{-\beta_{n}^{2}}{u} \int_{0}^{x} k_{z}\left(x^{\prime}\right) d x^{\prime}}\left(a_{n} \sin \left(\beta_{n} z\right)+b_{n} \cos \left(\beta_{n} z\right)\right)
$$

Applying the condition (20), then Eq. (23) becomes:

$$
\begin{gathered}
\alpha_{n} e^{\frac{-\beta_{n}^{2}}{u} \int_{0}^{x} k_{z}\left(x^{\prime}\right) d z^{\prime}} \frac{\partial}{\partial z}\left(a_{n} \sin \left(\beta_{n} z\right)+b_{n} \cos \left(\beta_{n} z\right)\right)=0 \\
a_{n} \beta_{n} \cos \left(\beta_{n} z\right)-b_{n} \beta_{n} \sin \left(\beta_{n} z\right)=0
\end{gathered}
$$




$$
\text { At } z=0 \Rightarrow a_{n} \beta_{n}=0 \Rightarrow a_{n}=0
$$

The equation (19) becomes:

$$
Z_{n}(z)=b_{n} \cos \left(\beta_{n} z\right)
$$

Then equation (23) is written in the form:

$$
c_{y}(x, z)=\alpha_{n} e^{\frac{-\beta_{n}^{2}}{u} \int_{0}^{x} k_{z}\left(x^{\prime}\right) d x^{\prime}}\left(b_{n} \cos \left(\beta_{n} z\right)\right)
$$

Applying the condition (21) then Eq. (28) can be written in the form: $\alpha_{n} e^{\frac{-\beta_{n}^{2}}{u} \int_{0}^{x} k_{z}\left(x^{\prime}\right) d x^{\prime}} \frac{d}{d z}\left(b_{n} \cos \left(\beta_{n} z\right)\right)=0$

$$
\begin{gathered}
\text { At } z=h \Rightarrow-b_{n} \beta_{n} \sin \left(\beta_{n} z\right)=0 \Rightarrow-b_{n} \beta_{n} \sin \left(\beta_{n} h\right)=0 \\
z=h \Rightarrow-b_{n} \beta_{n} \sin \left(\beta_{n} z\right)=0 \Rightarrow-b_{n} \beta_{n} \sin \left(\beta_{n} h\right)=0 \\
\Rightarrow \beta_{n} h=n \pi ; n=0,1,2, \ldots \ldots \ldots . \\
\quad \Rightarrow \beta_{n}=\frac{n \pi}{h} \quad \text { Then, } \\
Z_{n}(z)=b_{n} \cos \left(\frac{n \pi}{h} z\right) \text { at } \quad n=0 \Rightarrow \beta_{0}=0
\end{gathered}
$$

We can write the general solution in the form:

$$
C_{y}(x, z)=X_{o}(x) Z_{o}(z)+\sum_{n=1}^{\infty} X_{n}(x) Z_{n}(z)
$$

Eq. (30) can be written in the form:

$$
\begin{gathered}
C_{y}(x, z)=\alpha_{0} M+\sum_{n=1}^{\infty} X_{n}(x) Z_{n}(z) \\
c_{y}(x, z)=\alpha_{0} M+\sum_{n=1}^{\infty} b_{n} \cos \left(\beta_{n} z\right) \alpha_{n} e^{\frac{-\beta_{n}^{2}}{u} \int_{0}^{x} k_{z}\left(x^{\prime}\right) d x^{\prime}} \\
\text { Let } \quad R_{0}=\alpha_{0} M, b_{n} \alpha_{n}=R_{n} \\
c_{y}(x, z)=R_{0}+\sum_{n=1}^{\infty} R_{n} \cos \left(\frac{n \pi}{h} z\right) e^{\frac{-n^{2} \pi^{2}}{h^{2} u} \int_{0}^{x} k_{z}\left(x^{\prime}\right) d x^{\prime}}
\end{gathered}
$$

Using condition (22), one can get:

$$
\begin{gathered}
R_{0}+\sum_{n=1}^{\infty} R_{n} \cos \left(\frac{n \pi}{h} z\right) e^{\frac{-n^{2} \pi^{2}}{h^{2} u} \int_{0}^{x} k_{z}\left(x^{\prime}\right) d x^{\prime}}=\frac{Q}{u} \delta\left(z-h_{s}\right) \\
R_{0}+\sum_{n=1}^{\infty} R_{n} \cos \left(\frac{n \pi}{h} z\right) e^{\frac{-n^{2} \pi^{2}}{h^{2} u} \int_{0}^{x} k_{z}\left(x^{\prime}\right) d x^{\prime}}=\frac{Q}{u} \delta\left(z-h_{s}\right)
\end{gathered}
$$

Integrating with respect to $z$ from $z=0$ to $z=h$

$$
\begin{aligned}
\int_{0}^{h} R_{0} d z+\sum_{n=1}^{\infty} R_{n} \int_{0}^{h} \cos \left(\frac{n \pi}{h} z\right) d z & =\int_{0}^{h} \frac{Q}{u} \delta\left(z-h_{s}\right) d z \\
\Rightarrow R_{0} h+\sum_{n=1}^{\infty} \frac{h}{n \pi} R_{n} \sin (n \pi) & =\frac{Q}{u} \int_{0}^{h} \delta\left(z-h_{s}\right) d z
\end{aligned}
$$

But

$$
\sin (n \pi)=0 \Rightarrow R_{0} h=\frac{Q}{u}
$$

Equation (35) becomes:

$$
\frac{Q}{u h}+\sum_{n=1}^{\infty} R_{n} \cos \left(\frac{n \pi}{h} z\right)=\frac{Q}{u} \delta\left(z-h_{s}\right)
$$

Multiplying by $\cos \left(\frac{n \pi}{h} z\right)$ and integrating with respect to $z$ from $z=0$ to $\mathrm{h}$ :

$$
\begin{aligned}
& \frac{Q}{u h} \int_{0}^{h}\left(\cos \left(\frac{n \pi}{h} z\right)\right) d z+\sum_{n=1}^{\infty} R_{n} \int_{0}^{h} \cos ^{2}\left(\frac{n \pi}{h} z\right) d z=\frac{Q}{u} \int_{0}^{h} \delta\left(z-h_{s}\right) \cos \left(\frac{n \pi}{h} z\right) d z \\
& \frac{Q}{u h} \frac{h}{n \pi} \sin \left(\frac{n \pi}{h} z\right)+\sum_{n=1}^{\infty} R_{n} \int_{0}^{h} \frac{1}{2}\left(1+\cos \left(\frac{2 n \pi}{h} z\right)\right) d z=\frac{Q}{u} \int_{0}^{h} \delta\left(z-h_{s}\right) \cos \left(\frac{n \pi}{h} z\right) d z \\
& \text { then, } \frac{R_{n} h}{2}=\frac{Q}{u} \cos \left(\frac{n \pi}{h} h_{s}\right) \Rightarrow R_{n}=\frac{2 Q}{u h} \cos \left(\frac{n \pi}{h} h_{s}\right)
\end{aligned}
$$

The general solution becomes:

$c_{y}(x, z)=\frac{Q}{u h}\left(1+2 \sum_{n=1}^{\infty} \cos \left(\frac{n \pi h_{s}}{h}\right) \cos \left(\frac{n \pi z}{h}\right)\right) e^{\frac{-n^{2} \pi^{2}}{h^{2} u} \int_{0}^{x} k_{z}\left(x^{\prime}\right) d x^{\prime}}$

The value of the crosswind integrated concentration at ground put $z=0$ in Eq. (42), one can get:

$$
c_{y}(x, 0)=\frac{Q}{u h}\left(1+2 \sum_{n=1}^{\infty} \cos \left(\frac{n \pi h_{s}}{h}\right)\right) e^{\frac{-n^{2} \pi^{2}}{h^{2} u} \int_{0}^{x} k_{z}\left(x^{\prime}\right) d x^{\prime}}
$$

The value of the crosswind integrated concentration at ground source, put $h_{s}=0$ in Eq. (42), one can get:

$$
c_{y}(x, z)=\frac{Q}{u h}\left(1+2 \sum_{n=1}^{\infty} \cos \left(\frac{n \pi z}{h}\right)\right) e^{\frac{-n^{2} \pi^{2}}{h^{2} u} \int_{0}^{x} k_{z}\left(x^{\prime}\right) d x^{\prime}}
$$

Let the eddy diffusivity in the form:

$$
k_{z}(x)=\alpha u x
$$

Where " $\mathrm{u}$ " is the mean wind speed in $\mathrm{x}$-direction $(\mathrm{m} / \mathrm{s})$ described in region $0 \leq z \leq h, \mathrm{~h}$ is the mixing height and $\alpha$ is the turbulence intensity is taken in the form $\alpha=\left(\sigma_{w} / u\right)^{2}$ (54) where $\sigma_{w}$ is the standard deviation for vertical velocity. Then

$$
k_{z}(x)=\frac{\sigma_{w}^{2}}{u} x
$$

Substituting from equation (46) in equation (42), then the general equation becomes:

$$
c_{y}(x, z)=\frac{Q}{u h}\left(1+2 \sum_{n=1}^{\infty} \cos \left(\frac{n \pi h_{s}}{h}\right) \cos \left(\frac{n \pi z}{h}\right)\right) e^{\frac{-n^{2} \pi^{2} \sigma_{w}^{2} x^{2}}{2 h^{2} u^{2}}}
$$

put $z=0$ in Eq. (47), one can get: 


$$
c_{y}(x, 0)=\frac{Q}{u h}\left(1+2 \sum_{n=1}^{\infty} \cos \left(\frac{n \pi h_{s}}{h}\right)\right) e^{\frac{-n^{2} \pi^{2} \sigma_{v}^{2} x^{2}}{2 h^{2} u^{2}}}
$$

\section{Validation}

The used data was observed from the atmospheric diffusion experiments conducted at the northern part of Copenhagen, Denmark, under neutral and unstable conditions by [12] and [13]. Fig. (1) Shows that the predicted normalized crosswind integrated concentrations values of the present predicted model are good to the observed data as the Gaussian predicted model. Fig. (2) Shows that the present predicted data is nearer to the observed concentrations data than the predicted Gaussian model.

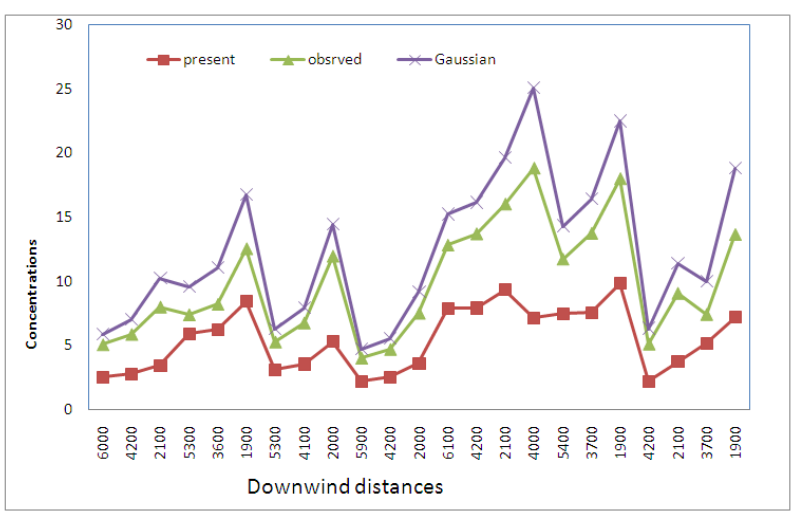

Figure 1. The variation of the two predicted and observed models via downwind distances.

From the two figures, we find that there is agreement between the predicted normalized crosswind integrated concentrations of present model with the observed normalized crosswind integrated concentrations as the predicted Gaussian model.

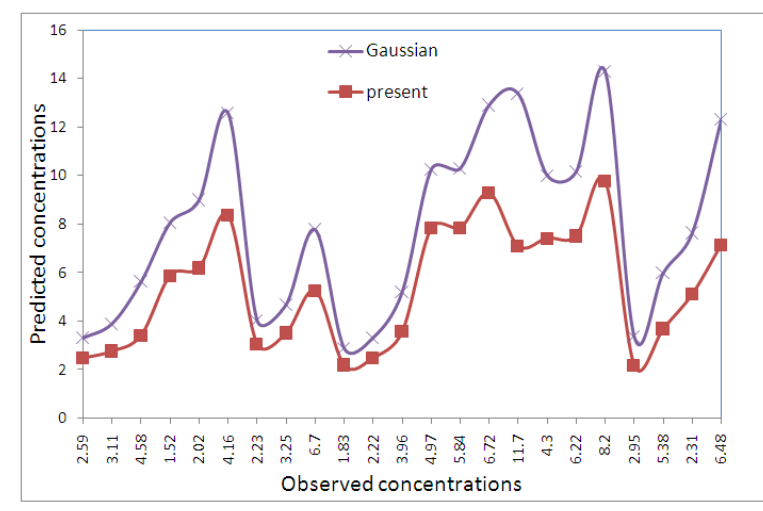

Figure 2. The variation between the predicted models and observed concentrations data.

\section{Statistical Method}

Now, the statistical method is presented and comparison between predicted and observed results will be offered by
[14]. The following standard statistical performance measures that characterize the agreement between prediction $(\mathrm{Cp}=\mathrm{Cpred} / \mathrm{Q})$ and observations $(\mathrm{Co}=\mathrm{Cobs} / \mathrm{Q})$ :

Normalized Mean Square Error (NMSE) $=\frac{\overline{\left(\mathrm{C}_{\mathrm{p}}-\mathrm{C}_{\mathrm{o}}\right)^{2}}}{\overline{\left(\mathrm{C}_{\mathrm{p}} \mathrm{C}_{\mathrm{o}}\right)}}$

Fractional Bias $(F B)=\frac{\left(\overline{C_{o}}-\overline{C_{p}}\right)}{\left[0.5\left(\overline{C_{o}}+\overline{C_{p}}\right)\right]}$

Correlation Coefficient (COR)

$$
=\frac{1}{N_{m}} \sum_{i=1}^{N_{m}}\left(C_{p i}-\overline{C_{p}}\right) \times \frac{\left(C_{o i}-\overline{C_{o}}\right)}{\left(\sigma_{p} \sigma_{o}\right.}
$$

Factor of Two $(F A C 2)=0.5 \leq \frac{C_{p}}{C_{o}} \leq 2.0$

Where $\sigma_{\mathrm{p}}$ and $\sigma_{\mathrm{o}}$ are the standard deviations of $\mathrm{C}_{\mathrm{p}}$ and Co respectively. Here the over bars indicate the average over all measurements. A perfect model would have the following idealized performance: $\mathrm{NMSE}=\mathrm{FB}=0$ and $\mathrm{COR}=1.0$.

Table 1. Comparison between our two predicted models according to standard statistical Performance measure.

\begin{tabular}{ccccc}
\hline Models & NMSE & FB & COR & FAC2 \\
\hline Present model & 0.22 & -0.19 & 0.60 & 1.38 \\
Gaussian model & 0.58 & 0.58 & 0.80 & 0.59 \\
\hline
\end{tabular}

From the statistical method (Table 1), we find that the two models are inside a factor of two with observed data. Regarding to NMSE and FB, the present predicted model is better good with observed data than the Gaussian model. The correlation of present predicated model equals $(0.60)$ and Gaussian model equals (0.80).

\section{Conclusions}

The predicted crosswind integrated concentrations of the two predicted models are inside a factor of two with observed concentration data. One finds that there is agreement between the present predicted normalized crosswind integrated concentrations model with the observed normalized crosswind integrated concentrations than the Gaussian predicted model with respect to normalized square error and fraction bias (NMSE and FB). The correlation of present predicated model equals $(0.60)$ and Gaussian model equals (0.80).

\section{References}

[1] Demuth, C. "A contribution to the analytical steady solution of the diffusion equation" Atmos. Environ, 1 2, 1255(1978).

[2] Tirabassi, T., Tagliazucca, M., Zannetti, P., KAPPAG, "A non-Gaussian plume dispersion model" JAPCA 36, 592-596, (1986).

[3] Lin, J.S. and Hildemann L.M., "generalized mathematical schemes to analytical solve the atmospheric diffusion equation with dry deposition, Atmos. Environ". 31, 59-(1997). 
[4] John M. Stockie, The Mathematics of atmospheric dispersion molding. Society for Industrial and Applied Mathematics. Vol. 53.No.2 pp. 349-372, (2011).

[5] Van Ulden A.P., Hotslag, A. A. M., and "Estimation of atmospheric boundary layer parameters for diffusion applications Journal of Climate and Applied Meteorology 24, 1196- 1207(1978).

[6] Pasquill, F., Smith, F.B., "Atmospheric Diffusion $3^{\text {rd }}$ edition". Wiley, New York, USA,(1983).

[7] Seinfeld, J.H" Atmospheric Chemistry and physics of Air Pollution”. Wiley, New York, (1986).

[8] Sharan, M., Singh, M.P., Yadav, A.K," Mathematical model for atmospheric dispersion in low winds with eddy diffusivities as linear functions of downwind distance". Atmospheric Environment 30, 1137-1145, (1996).

[9] Essa K.S.M., and E,A.Found ,"Estimated of crosswind integrated Gaussian and Non-Gaussian concentration by using different dispersion schemes". Australian Journal of Basic and Applied Sciences, 5(11): 1580-1587, (2011).

[10] Arya, S. P "Modeling and parameterization of near -source diffusion in weak wind" J. Appl .Met. 34, 1112-1122. (1995).

[11] Essa K. S. M., Maha S. EL-Qtaify" Diffusion from a point source in an urban Atmosphere" Meteol. Atmo, Phys., 92, 95-101, (2006)

[12] Gryning S. E., and Lyck E "Atmospheric dispersion from elevated sources in an urban area: Comparison between tracer experiments and model calculations", J. Climate Appl. Meteor.,23, pp. 651-660., (1984).

[13] Gryning S.E., Holtslag, A.A.M., Irwin, J.S., Sivertsen, B., "Applied dispersion modeling based on meteorological scaling parameters", Atoms. Environ. 21 (1), 79-89 (1987).

[14] Hanna S. R., 1989, "confidence limit for air quality models as estimated by bootstrap and Jackknife resembling methods", Atom. Environ. 23, 1385-139 